Short Communication

\title{
Long-term outcomes with agalsidase alfa enzyme replacement therapy: Analysis using deconstructed composite events
}

\author{
Michael Beck ${ }^{\mathrm{a}, *}$, Derralynn Hughes ${ }^{\mathrm{b}}$, Christoph Kampmann ${ }^{\mathrm{a}}$, Guillem Pintos-Morell ${ }^{\mathrm{c}}$, \\ Uma Ramaswami $^{\mathrm{b}}$, Michael L. West ${ }^{\mathrm{d}}$, Roberto Giugliani ${ }^{\mathrm{e}}$

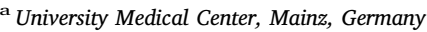 \\ ${ }^{\mathrm{b}}$ Royal Free London NHS Foundation Trust, University College London, London, UK \\ ' University Hospital and Research Institute "Germans Trias i Pujol", Badalona, Universitat Autònoma de Barcelona, Barcelona, Spain \\ ${ }^{\mathrm{d}}$ Department of Medicine, Dalhousie University, Halifax, NS, Canada \\ ${ }^{\mathrm{e}}$ Medical Genetics Service HCPA/Department of Genetics UFRGS and INAGEMP, Porto Alegre, Brazil
}

A R T I C L E I N F O

\section{Keywords:}

Fabry disease

Enzyme replacement therapy

Agalsidase alfa

Long-term effectiveness

\begin{abstract}
A B S T R A C T
This is a retrospective analysis of Fabry Outcome Survey data from children/adults $(n=677)$ receiving agalsidase alfa enzyme replacement therapy for a median of 3 years, examining cerebrovascular, cardiac, and renal morbidity endpoints separately. Cardiac events occurred at younger ages than cerebrovascular or renal events, cerebrovascular events were more frequent in females than males, and males were more likely to experience cardiac and renal events at a younger age than females.
\end{abstract}

\section{Introduction}

Fabry disease (OMIM 301500) is a rare inherited lysosomal storage disorder associated with reduced activity of $\alpha$-galactosidase A (EC 3.2.1.22) [1] and characterized by significant morbidity, including renal impairment, cardiomyopathy, and cerebrovascular events [2-5].

In a recent analysis, we retrospectively compared long-term composite morbidity outcomes in patients from the Fabry Outcome Survey (FOS; sponsored by Shire) who were receiving agalsidase alfa enzyme replacement therapy (ERT) with published outcomes in untreated patients with Fabry disease [6]. Long-term ERT appeared to slow the progression of renal impairment and cardiomyopathy, and composite morbidity events occurred later in treated patients. Our current objective was to further examine and describe long-term morbidity rates separately by cerebrovascular, cardiac, and renal event origin in patients from FOS receiving agalsidase alfa ERT.

\section{Materials and methods}

Data were extracted from FOS for the period from database inception in 2001 to November 2012. Patient disposition is shown in Fig. A.1. The FOS registry collects standardized information from patients who are managed at participating centers and provide signed informed consent. FOS has been approved by the ethics committee/institutional review board of all participating centers and all procedures were in accordance with the Declaration of Helsinki of 1975, revised in 2013.

Members of the FOS evaluable treated cohort $(n=677)$ had all received agalsidase alfa ERT, including 86 children and 591 adults who initiated treatment before or after FOS entry. Patients who had received a kidney transplant or initiated dialysis before starting ERT were excluded, as were those who had received another ERT before FOS entry; 66 patients who left FOS were censored from the date they left.

Estimated glomerular filtration rate (eGFR) was calculated in adults (patients $\geq 18$ years of age) only, using a four-variable Modification of Diet in Renal Disease equation [7]. Baseline left ventricular hypertrophy (LVH) was defined by left ventricular mass index (LVMI) $>48$ $\mathrm{g} / \mathrm{m}^{2.7}$ in females or $>50 \mathrm{~g} / \mathrm{m}^{2.7}$ in males. Baseline data regarding proteinuria and Fabry phenotype were not available.

Morbidity as age at first event was examined separately for cerebrovascular, cardiac, and renal events. Cerebrovascular events included stroke, transient ischemic attack, or prolonged reversible ischemic neurologic deficit. Cardiac events included myocardial infarction, cardiac surgery, arrhythmia, angina, or heart failure. Renal events included renal transplant, dialysis, or $\geq 33 \%$ increase (in two consecutive values) from baseline in serum creatinine. Kaplan-Meier analyses were conducted for age at morbidity (from birth to first event) with censoring at last visit.

\footnotetext{
Abbreviations: eGFR, estimated glomerular filtration rate; ERT, enzyme replacement therapy; FOS, Fabry Outcome Survey; LVH, left ventricular hypertrophy; NE, not estimable * Corresponding author at: Children's Hospital, University Medical Center, J.G. University Mainz, Langenbeckstrasse 1, 55101 Mainz, Germany.

E-mail address: Michael.Beck@unimedizin-mainz.de (M. Beck).
} 
A

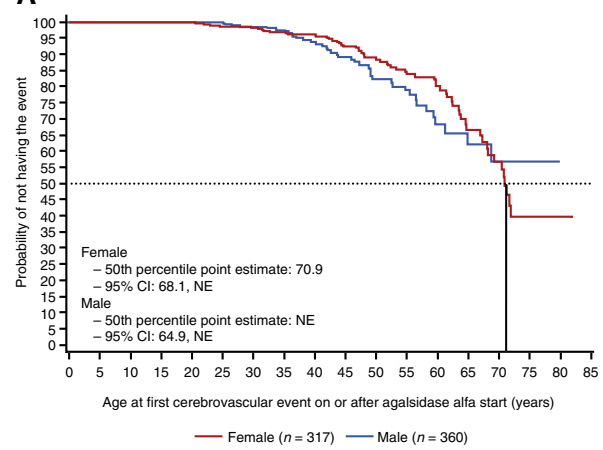

B

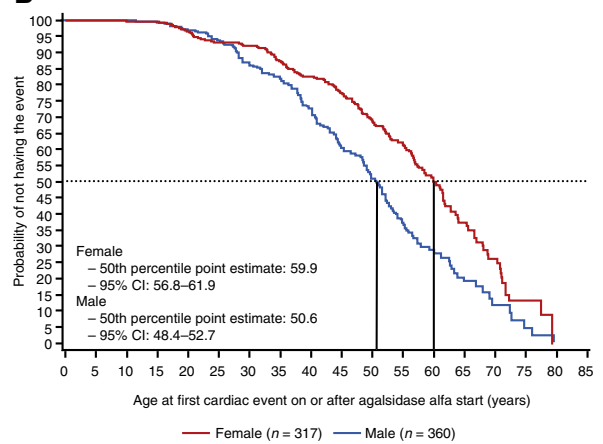

C

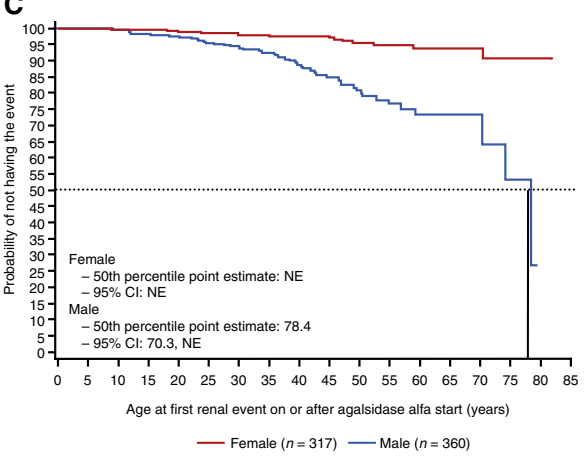

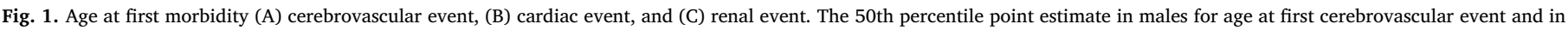
females for age at first renal event was not estimable (NE) because of the small number of events.

\section{Results}

Baseline demographic and clinical characteristics are shown in Table A.1. Among the 677 patients ( 360 male, 317 female) in the overall FOS evaluable treated cohort, median (range) age at first Fabry disease symptom was $12(0-72)$ years and median (range) age at diagnosis was $31(0-79)$ years. Median (range) time on treatment (time from treatment initiation to data extraction) was $3(0-14)$ years. At baseline, $63 / 447$ (14.1\% of adult patients with a baseline eGFR value available) had eGFR $<60 \mathrm{~mL} / \mathrm{min} / 1.73 \mathrm{~m}^{2}$ and $143 / 330(43.3 \%$ of patients with a baseline LVMI value available) had LVH.

In the overall FOS evaluable treated cohort, the number and percentage of patients who did not experience an event during follow-up was: cerebrovascular, 578 (85.4\%); cardiac, 378 (55.8\%); renal, 614 (90.7\%); any morbidity event, 326 (48.2\%; Table A.2). The KaplanMeier point estimate $(95 \% \mathrm{CI})$ for median age in years at first event was: cerebrovascular, 70.9 (68.1, not estimable [NE; because of the small number of events]) in females and $\mathrm{NE}(64.9, \mathrm{NE})$ in males (Fig. 1A); cardiac, 59.9 (56.8-61.9) in females and 50.6 (48.4-52.7) in males (Fig. 1B); renal, NE (NE, NE) in females and 78.4 (70.3, NE) in males (Fig. 1C).

Among female patients, the probability of not having a renal event was relatively high even at advanced ages; for example, at age 75 years, the probability of not having a renal event was $\sim 90 \%$ in women and $\sim 55 \%$ in men.

\section{Discussion}

The current analysis is an extension of our retrospective analysis [6], which found that agalsidase alfa ERT appeared to slow the progression of renal impairment and LVH, and composite morbidity events (including death) occurred later, in FOS patients receiving agalsidase alfa ERT compared with published findings in untreated patients with Fabry disease. The current work represents a purely descriptive analysis of the status of treated patients; because of the lack of external comparator groups, no comparison of agalsidase alfa treated and untreated patients was possible. Our findings are consistent with other published analyses showing stable or improved cardiac or renal parameters in patients receiving ERT with agalsidase alfa [3,8-12]. Compared with similar analyses in the published literature [8,13-15], our analysis is based on the largest dataset of clinical events in the long-term treatment of Fabry disease to date. However, the number of renal events recorded in the FOS database was still relatively small, limiting the analysis of renal events and indicating that renal function is well maintained over years of ERT.

Although the current analysis showed a somewhat higher proportion of patients with an event than that reported by Germain et al. [8], the definition of events was not comparable between these two analyses. For consistency with our previous analysis [6], which compared treated patients from FOS with untreated patients in the published literature [16], the definition of events was broader in our FOS analysis than in the analysis of Germain et al. [8], and therefore some of these events were less severe (e.g., transient ischemic attack, prolonged reversible ischemic neurologic deficit). In contrast, Germain et al. focused on severe clinical events, which were defined as "chronic dialysis, kidney transplant, myocardial infarction, congestive heart failure, major cardiac procedures (i.e., implantation of a balloon pump, cardioverter-defibrillator or first pacemaker; or bypass surgery), stroke and death" [8].

Several limitations of our analysis should be noted. This was a retrospective analysis of FOS registry data and the FOS patients were not randomly selected, which may contribute to selection and/or ascertainment bias. In general, registry analyses have a higher probability of patient loss from the analysis because of the relative lack of data completeness compared with randomized controlled trials. Comparisons were not made with untreated patients from this registry; consequently, no definitive conclusions can be made regarding effectiveness of ERT. FOS lacks a robust cohort of untreated patients for comparison with treated patients. In general, untreated patients in FOS have less severe disease and undergo fewer follow-up assessments; thus, fewer outcome data are available [17]. Although LVMI is not itself a clinical endpoint, LVH has been associated with both cardiac and renal clinical outcomes in patients with Fabry disease [17,18]. A potential source of bias is the censoring of patients who discontinued agalsidase alfa ERT.

Over a median period of 3 years of agalsidase alfa ERT in this registry population of patients with long-standing Fabry disease, cardiac events occurred more frequently and at younger ages than cerebrovascular or renal events. Cerebrovascular events were more frequent in females than in males. Males were more likely to experience cardiac and renal events at a younger age than females. It is possible that the presence of cardiac and renal events in males, and their subsequent treatment, may decrease the incidence of later cerebrovascular events relative to the incidence in females. Although we analyzed a cohort of FOS patients with a median age of 46.2 years, one must also consider that, from a US population perspective, the 10-year risk of stroke is higher in middle-aged women aged 55 years than middle-aged men aged 55 years [19]. The main determinant of stroke risk other than advancing age is blood pressure [19], which an earlier analysis showed to be uncontrolled in over half of untreated FOS patients at baseline before declining after 2 years of agalsidase alfa ERT [20]. Analysis of 10 -year outcomes in patients receiving agalsidase alfa ERT using deconstructed composite events will shed more light on these observations.

\section{Role of the funding source}

The Fabry Outcome Survey is sponsored by Shire Human Genetic 
Therapies, Inc. Shire International $\mathrm{GmbH}$ provided funding for data collection and analysis, and provided funding to Excel Scientific Solutions for support in writing and editing this manuscript. Under the direction of the authors, Margit Rezabek, DVM, PhD, employee of Excel Scientific Solutions, provided writing assistance for this publication. Editorial assistance in formatting, proofreading, copyediting, and fact checking was also provided by Excel Scientific Solutions. Jaco Botha, $\mathrm{MSc}$, of Shire International $\mathrm{GmbH}$, and Svetlana Bizjajeva, $\mathrm{PhD}$, formerly of Shire International $\mathrm{GmbH}$, provided statistical support and reviewed and edited the manuscript for scientific accuracy. Sylvain Larroque, MSc, formerly of Shire International $\mathrm{GmbH}$, planned and conducted statistical analyses.

\section{Contributions}

All authors reviewed and revised early and final drafts of the manuscript and were fully responsible for editorial decisions related to this manuscript.

\section{Conflict of interest}

Michael Beck has received unrestricted grants, travel support, and honoraria from Actelion, BioMarin, Genzyme, Shire, and Synageva.
Derralynn Hughes has received travel/research grants and honoraria for speaking/consulting from Amicus, Protalix, Sanofi-Genzyme, and Shire. Christoph Kampmann has received research support from BioMarin and Shire; consulting/speaker fees from Amicus, BioMarin, Gore, and Shire; participated in clinical studies sponsored by Actelion, BioMarin, Genzyme, Gore, and Shire; and served on advisory committees for BioMarin and Shire. Guillem Pintos-Morell has received travel grants/honoraria for consulting/lectures from Alexion, BioMarin, and Shire. Uma Ramaswami has received travel/research grants and honoraria for lectures/consulting from Amicus, Alexion, Genzyme, Protalix, and Shire. Michael L. West has received research grants, consulting/ speaker fees, and/or travel support from Actelion, Alexion, Amicus, AvroBio, Excelsior, GlaxoSmithKline, Sanofi-Genzyme, Shire, and Sumitomo. Roberto Giugliani has received consulting fees from Amicus, BioMarin, Genzyme, Shire, and Synageva/Alexion; participated in clinical trials sponsored by Amicus, BioMarin, Genzyme, GlaxoSmithKline, Shire, and Synageva/Alexion; and assisted in the design of and/or participated in clinical studies using products manufactured by Actelion, BioMarin, Genzyme, and Shire.

\section{Funding}

This work was supported by Shire International GmbH.

\section{Appendix A}

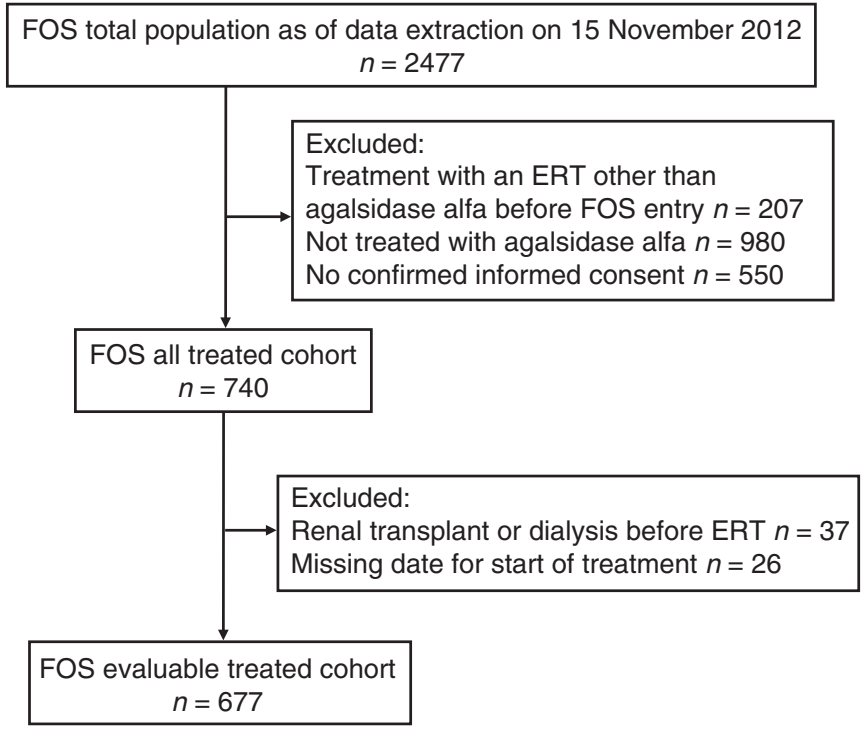

Fig. A.1. Disposition of patients. ERT, enzyme replacement therapy; FOS, Fabry Outcome Survey.

Table A. 1

Demographic and clinical characteristics of patients in the FOS evaluable treated cohort.

\begin{tabular}{|c|c|c|c|}
\hline Race, $n(\%)$ & $n=358$ & $n=311$ & $n=669$ \\
\hline Asian & $58(16.2)$ & $40(12.9)$ & $98(14.6)$ \\
\hline Other & $6(1.7)$ & $9(2.9)$ & $15(2.2)$ \\
\hline Age at data extract (years) & $n=360$ & $n=317$ & $n=677$ \\
\hline
\end{tabular}


Mean (SD)

Median (IQR) [range]

Age at first symptom (years)

Mean (SD)

Median (IQR) [range]

Age at diagnosis (years)

Mean (SD)

Median (IQR) [range]

Age at treatment initiation (years)

Mean (SD)

Median (IQR) [range]

Time from initiation to last date of treatment (years)

Mean (SD)

Median (IQR) [range]

Median (IQR) baseline systolic BP (mmHg)

Median (IQR) baseline diastolic BP (mmHg)

Median (IQR) baseline total FOS-MSSI score

Baseline eGFR category $<60 \mathrm{~mL} / \mathrm{min} / 1.73 \mathrm{~m}^{2}, n(\%)$

Baseline LVH, $n$ (\%)

$$
\begin{aligned}
& 40.8(16.2) \\
& 38.7 \\
& (28.2-53.5) \\
& {[8.6-82.4]} \\
& n=287 \\
& 14.3(14.4) \\
& 10.0 \\
& (6.0-14.0) \\
& {[0.0-70.0]} \\
& n=341 \\
& 27.1(17.8) \\
& 24.0 \\
& (13.5-37.8) \\
& {[0.0-79.0]} \\
& n=360 \\
& 33.8(16.1) \\
& 31.8 \\
& (20.8-45.5) \\
& {[2.5-79.5]} \\
& n=360 \\
& 4.3(3.7) \\
& 3.2(1.0-7.0) \\
& {[0.0-13.8]}
\end{aligned}
$$

\begin{tabular}{|c|c|}
\hline $50.6(16.0)$ & $45.4(16.8)$ \\
\hline 52.3 & 46.2 \\
\hline (40.5-62.4) & $(31.9-58.2)$ \\
\hline [12.0-84.6] & {$[8.6-84.6]$} \\
\hline$n=230$ & $n=517$ \\
\hline $24.9(18.5)$ & $19.0(17.2)$ \\
\hline 18.0 & 12.0 \\
\hline$(9.8-40.0)$ & $(7.0-27.3)$ \\
\hline$[0.0-72.0]$ & {$[0.0-72.0]$} \\
\hline$n=301$ & $n=642$ \\
\hline 39.1 (17.4) & 32.7 (18.6) \\
\hline 42.0 & 31.0 \\
\hline$(27.0-51.0)$ & $(16.0-48.0)$ \\
\hline$[2.0-78.0]$ & {$[0.0-79.0]$} \\
\hline$n=317$ & $n=677$ \\
\hline $44.6(16.2)$ & $38.9(17.0)$ \\
\hline 47.0 & 39.3 \\
\hline$(34.0-56.4)$ & $(25.2-52.1)$ \\
\hline [4.4-79.2] & {$[2.5-79.5]$} \\
\hline$n=317$ & $n=677$ \\
\hline $3.9(3.2)$ & $4.1(3.5)$ \\
\hline $2.9(1.2-6.3)$ & 3.0 \\
\hline \multirow[t]{2}{*}{ [0.0-11.9] } & $(1.1-6.7)$ \\
\hline & {$[0.0-13.8]$} \\
\hline$n=244$ & $n=524$ \\
\hline 123.0 & 121.0 \\
\hline (112.0-136.- & (110.0-13- \\
\hline 0) & $5.0)$ \\
\hline$n=244$ & $n=524$ \\
\hline 73.0 & 70.5 \\
\hline$(62.5-80.0)$ & $(64.0-80.0)$ \\
\hline$n=304$ & $n=648$ \\
\hline 16.5 & 16.0 \\
\hline (8.8-25.3) & $(8.5-24.5)$ \\
\hline$n=218$ & $n=447$ \\
\hline 32 (14.7) & $63(14.1)$ \\
\hline$n=176$ & $n=330$ \\
\hline $82(46.6)$ & $143(43.3)$ \\
\hline
\end{tabular}

$n=280$
120.0
$(110.0-131 .-$
$5)$
$n=280$
70.0
$(64.0-80.0)$
$n=344$
15.0
$(8.0-23.5)$
$n=229$
$31(13.5)$
$n=154$
$61(39.6)$

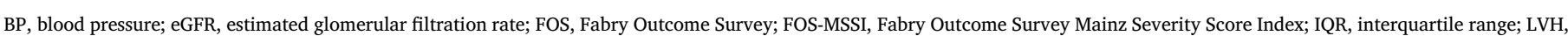
left ventricular hypertrophy.

Table A.2

Number (percentage) [95\% CI] of patients without a morbidity event.

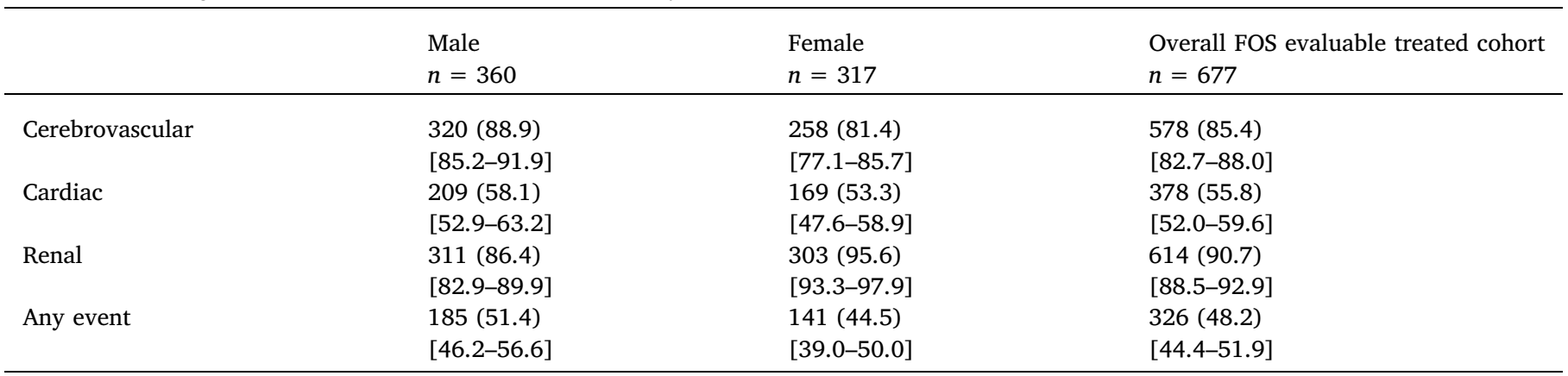

FOS, Fabry Outcome Survey.

\section{References}

[1] S.C. Garman, D.N. Garboczi, The molecular defect leading to Fabry disease: structure of human alpha-galactosidase, J. Mol. Biol. 337 (2004) 319-335.

[2] M. Branton, R. Schiffmann, J.B. Kopp, Natural history and treatment of renal involvement in Fabry disease, J. Am. Soc. Nephrol. 13 (2002) S139-S143.

[3] C. Kampmann, A. Linhart, F. Baehner, T. Palecek, C.M. Wiethoff, E. Miebach,
C. Whybra, A. Gal, J. Bultas, M. Beck, Onset and progression of the Anderson-Fabry disease related cardiomyopathy, Int. J. Cardiol. 130 (2008) 367-373.

[4] K.D. MacDermot, A. Holmes, A.H. Miners, Natural history of Fabry disease in affected males and obligate carrier females, J. Inherit. Metab. Dis. 24 (2001) 13-14 (discussion 11-12).

[5] A. Mehta, J.T. Clarke, R. Giugliani, P. Elliott, A. Linhart, M. Beck, G. SunderPlassmann, FOS Investigators, Natural course of Fabry disease: changing pattern of causes of death in FOS - Fabry Outcome Survey, J. Med. Genet. 46 (2009) 548-552. 
[6] M. Beck, D. Hughes, C. Kampmann, S. Larroque, A. Mehta, G. Pintos-Morell, U. Ramaswami, M. West, A. Wijatyk, R. Giugliani, the Fabry Outcome Survey Study Group, Long-term effectiveness of agalsidase alfa enzyme replacement in Fabry disease: a Fabry Outcome Survey analysis, Mol. Genet. Metab. Rep. 3 (2015) 21-27.

[7] K/DOQI clinical practice guidelines for chronic kidney disease: evaluation, classification, and stratification, Am. J. Kidney Dis. 39 (2002) S1-S266.

[8] D.P. Germain, J. Charrow, R.J. Desnick, N. Guffon, J. Kempf, R.H. Lachmann, R. Lemay, G.E. Linthorst, S. Packman, C.R. Scott, S. Waldek, D.G. Warnock, N.J. Weinreb, W.R. Wilcox, Ten-year outcome of enzyme replacement therapy with agalsidase beta in patients with Fabry disease, J. Med. Genet. 52 (2015) 353-358.

[9] C. Kampmann, A. Perrin, M. Beck, Effectiveness of agalsidase alfa enzyme replacement in Fabry disease: cardiac outcomes after 10 years' treatment, Orphanet J. Rare Dis. 10 (2015) 125.

[10] A. Schwarting, F. Dehout, S. Feriozzi, M. Beck, A. Mehta, G. Sunder-Plassmann, European FOS Investigators, Enzyme replacement therapy and renal function in 201 patients with Fabry disease, Clin. Nephrol. 66 (2006) 77-84.

[11] M. Beck, R. Ricci, U. Widmer, F. Dehout, A.G. de Lorenzo, C. Kampmann, A. Linhart, G. Sunder-Plassmann, G. Houge, U. Ramaswami, A. Gal, A. Mehta, Fabry disease: overall effects of agalsidase alfa treatment, Eur. J. Clin. Investig. 34 (2004) 838-844.

[12] S. Feriozzi, J. Torras, M. Cybulla, K. Nicholls, G. Sunder-Plassmann, M. West, The effectiveness of long-term agalsidase alfa therapy in the treatment of Fabry nephropathy, Clin. J. Am. Soc. Nephrol. 7 (2012) 60-69.

[13] M. Banikazemi, J. Bultas, S. Waldek, W.R. Wilcox, C.B. Whitley, M. McDonald, R. Finkel, S. Packman, D.G. Bichet, D.G. Warnock, R.J. Desnick, Fabry Disease Clinical Trial Study Group, Agalsidase-beta therapy for advanced Fabry disease: a randomized trial, Ann. Intern. Med. 146 (2007) 77-86.
[14] J.H. Kim, B.H. Lee, J. Hyang Cho, E. Kang, J.H. Choi, G.H. Kim, H.W. Yoo, Longterm enzyme replacement therapy for Fabry disease: efficacy and unmet needs in cardiac and renal outcomes, J. Hum. Genet. 61 (2016) 923-929.

[15] M. Lenders, B. Schmitz, J. Stypmann, T. Duning, S.M. Brand, C. Kurschat, E. Brand, Renal function predicts long-term outcome on enzyme replacement therapy in patients with Fabry disease, Nephrol. Dial. Transplant. (2016) (pii: gfw334. [Epub ahead of print]).

[16] R. Schiffmann, D.G. Warnock, M. Banikazemi, J. Bultas, G.E. Linthorst, S. Packman, S.A. Sorensen, W.R. Wilcox, R.J. Desnick, Fabry disease: progression of nephropathy, and prevalence of cardiac and cerebrovascular events before enzyme replacement therapy, Nephrol. Dial. Transplant. 24 (2009) 2102-2111.

[17] A. Mehta, M. Beck, P. Elliott, R. Giugliani, A. Linhart, G. Sunder-Plassmann, R. Schiffmann, F. Barbey, M. Ries, J.T. Clarke, Fabry Outcome Survey Investigators, Enzyme replacement therapy with agalsidase alfa in patients with Fabry's disease: an analysis of registry data, Lancet 374 (2009) 1986-1996.

[18] A. Linhart, C. Kampmann, J.L. Zamorano, G. Sunder-Plassmann, M. Beck, A. Mehta, P.M. Elliott, European FOS Investigators, Cardiac manifestations of Anderson-Fabry disease: results from the international Fabry outcome survey, Eur. Heart J. 28 (2007) 1228-1235.

[19] S. Seshadri, A. Beiser, M. Kelly-Hayes, C.S. Kase, R. Au, W.B. Kannel, P.A. Wolf, The lifetime risk of stroke: estimates from the Framingham Study, Stroke 37 (2006) 345-350.

[20] J. Kleinert, F. Dehout, A. Schwarting, A.G. de Lorenzo, R. Ricci, C. Kampmann, M. Beck, U. Ramaswami, A. Linhart, A. Gal, G. Houge, U. Widmer, A. Mehta, G. Sunder-Plassmann, Prevalence of uncontrolled hypertension in patients with Fabry disease, Am. J. Hypertens. 19 (2006) 782-787. 